\title{
LOW INSERTION LOSS PACKAGED AND FIBER-CONNECTORIZED SI SURFACE-MICROMACHINED REFLECTIVE OPTICAL SWITCH
}

\author{
V. Aksyuk, B. Barber, C.R. Giles, R. Ruel, L. Stulz, and D. Bishop \\ Bell Laboratories, Lucent Technologies, 700 Mountain Ave. \\ Murray Hill, NJ 07974
}

\begin{abstract}
A Si surface micromachined single mode fiber optical switch is using a novel vertical actuator to interpose a gold coated selfassembling silicon vane in a fiber gap to achieve $0.81 \mathrm{~dB}$ minimum insertion loss in the transmit state with $38-80 \mathrm{~dB}$ transmission isolation and $2.15 \mathrm{~dB}$ return loss in the reflection state, all in the $1550 \mathrm{~nm}$ wavelength region. The minimum actuation voltage is $4 \mathrm{~V}$ with virtually no power consumption. Rise and fall times as low as $50 \mu$ s are obtained with $20 \mathrm{~V}$ drive voltage.
\end{abstract}

\section{INTRODUCTION}

Conventional opto-mechanical fiberoptic switches have low insertion loss, high contrast ratio, wide optical bandwidth and low polarization dependence. However they are relatively bulky, expensive and slow. Micromachined switches combining the performance of bulk mechanical switches with small size, low cost and moderate speed are excellent candidates for a variety of applications, including reconfiguration switches in optical crossconnects and in WDM add/drop multiplexers. A number of devices have been reported utilizing bulk [1], surface micromachining $[2,3]$ and other fabrication techniques [4]. However, of those suitable for lightwave applications some require unique processes, others need complex manual assembly, while yet others have large actuation voltages or consume unacceptably high power.

We used a relatively simple commercially available $\mathrm{Si}$ surface micromachining process to build a set of MEMS optical switches featuring excellent optical performance, low actuation voltage, ultra-low power consumption, small size, moderate speed, no manual assembly of micromechanical structures and potentially low cost.

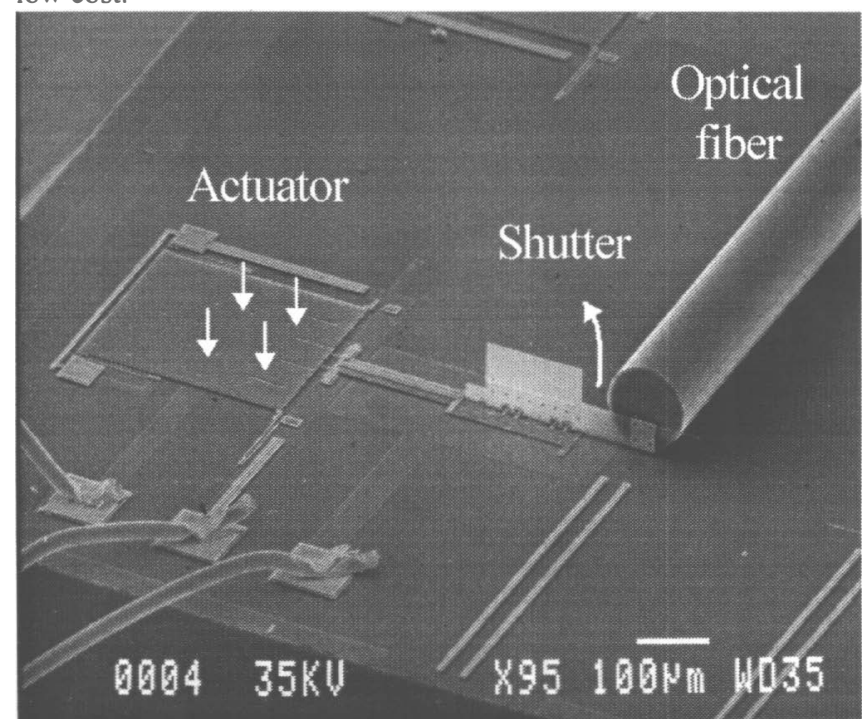

Figure 1. MEMS optical switch. For clarity the second optical fiber is not shown.

\section{DESIGN AND FABRICATION}

Fig. 1 is an SEM micrograph of one of our switches. The three layer Si surface micromachining Multi-User MEMS Process (MUMPS) available through MCNC was used for fabrication [5]. Switches were built with one fixed and two structural polisilicon layers, $0.5,2$ and $1.5 \mu \mathrm{m}$ thick. Si nitride was used to insulate the micromechanical structure from the wafer. Mirror surface was produced with $\mathrm{Cr}$-gold metallization. Phosphosilicon glass sacrificial layers were removed by etching in HF during the final release step. Each $2.5 \times 2.5 \mathrm{~mm}^{2}$ die was then epoxied in a ceramic 16 pin dual-in-line package (DIP) and single-mode optical fibers suitable for $1550 \mathrm{~nm}$ wavelength region were placed in microfabricated alignment rails with less then $20 \mu \mathrm{m}$ gap between the fiber faces. No active alignment was required to achieve low insertion loss. The fibers were fixed to the chip and package with a UV curable adhesive.

All our devices are two-port reflective switches consisting of a movable shutter connected to a novel electrostatic out-of-plane actuator capable of inserting the shutter in the light path between two fibers. The shutter is a polysilicon plate with a reflective gold layer deposited on one of its sides forming a mirror. The plate is connected to the actuator lever by means of hinges [6] and has to be rotated 90 degrees to its operating vertical position upon the release. While in earlier versions of the switch this assembly had to be done manually, later versions (Fig.2) include a self-assembly mechanism to perform this step automatically upon release. The mechanism uses energy stored in high tensile residual stress metal layer to produce vertical motion, which is then used to rotate and lock the shutter in its final position.

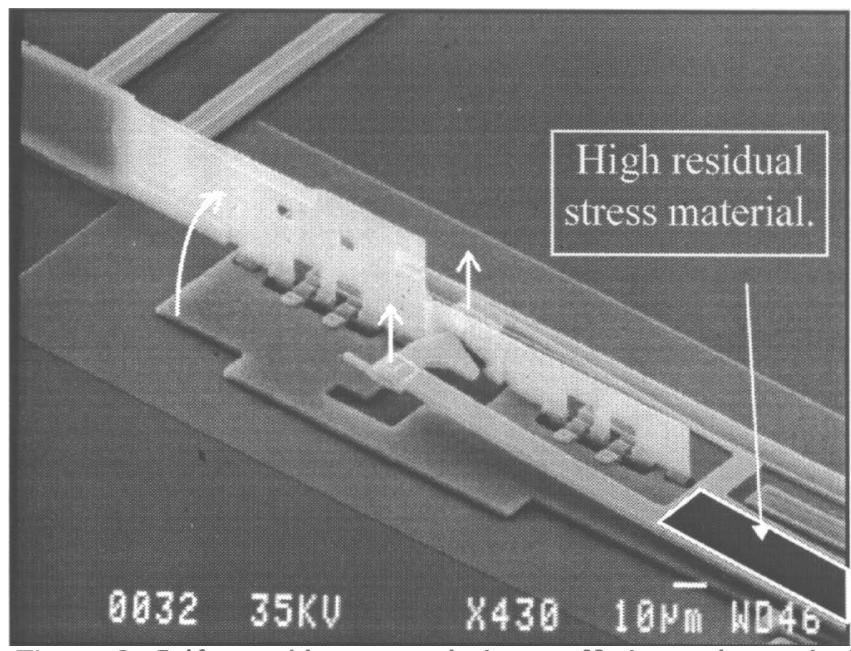

Figure 2. Self-assembling optical shutter. High tensile residual stress metal is deposited on a polysilicon beam anchored at one end. Upon release the metal-poly sandwich structure deforms, moving the free end of the beam upward. The lifting structure engages the cut in the hinged-plate shutter causing it to rotate 90 degrees into its operating position. 

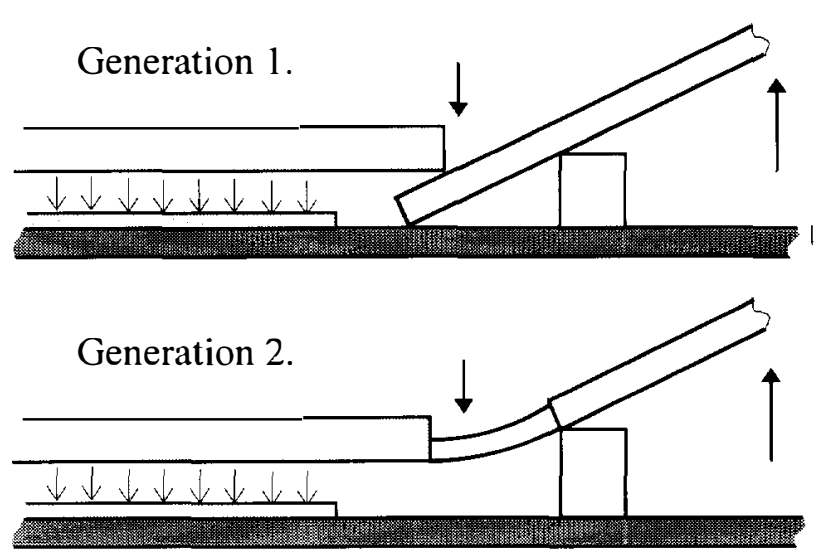

Figure 3. Simplified actuator schematic. In reality, the actuator plate is not rigid, resulting in a complicated zip lock - like capacitor closing. The resting posts are also not shown.

With zero volts applied to the actuator, the shutter edge is 5 to $10 \mu \mathrm{m}$ below the centers the two fiber cores, and the light is transmitted from one fiber into the other. The fiber faces are antireflection coated $(\mathrm{R} \approx 0.1 \%)$ to reduce unwanted backreflection and Fabry-Perot resonances. When voltage is applied, the shutter is moved upward into the light path, blocking transmission and reflecting the light back into the input fiber. A relatively large shutter displacement (more than $15 \mu \mathrm{m}$, determined by the $8 \mu \mathrm{m}$ mode diameter of the single mode telecommunication fiber used) is required to make a low insertion loss, high contrast ratio switch.

The schematic of the actuator is shown in Fig.3. A downward force is generated by an electrostatic actuator consisting of two initially parallel plates. A lever mechanism is then used to produce a large vertical displacement of the shutter The capacitor dimensions are ranging from $220 \times 170$ to $300 \times 350 \mu \mathrm{m}^{2}$ with a typical 0 -volt gap between the plates of $2.75 \mu \mathrm{m}$. The bottom plate is fixed and the top plate is suspended on a flexible support. To prevent the top plate from touching the bottom plate and shorting the capacitor, an array of $0.75 \mu \mathrm{m}$ tall polysilicon posts (dimples) are protruding from the bottom surface of the top plate. Holes are made in the bottom plate corresponding to the posts such that the posts touch down not on the bottom plate but on the insulating silicon nitride layer beneath it. The lever mechanism includes a 500 to $575 \mu \mathrm{m}$ long polysilicon beam capable of rotation around a horizontal pivot axis, providing the necessary mechanical advantage. In one version of the switch (Generation 1) the beam is attached to the substrate by weak torsional springs and engaged under the edge of the movable capacitor plate. In the other (Generation 2) it is connected to the plate by means of 2 thin polysilicon flexures measuring typically 1.5 by 2 by $18 \mu \mathrm{m}$ (Fig. 3 ). The size of the flexures is chosen to provide sufficient beam rotation angle for a given capacitor gap, while not requiring too high of a voltage to deform.

\section{RESULTS AND DISCUSSION}

In Fig. 4 the lever angle and corresponding shutter displacement are plotted as a function of voltage applied to the actuator. Because the moving plate of the actuator is only $1.5 \mu \mathrm{m}$ thick, and the mechanical stress is mostly concentrated at the point where the lever mechanism is connected to the plate, the deformation of the plate is significant and plays an important role during actuation. In fact, especially for stiff Generation 2 lever mechanisms, the actuator mostly works in a "zip-lock" mode as opposed to "parallel-plate" mode. As the voltage is increased, the part of the plate furthest from the lever moves down until the resting posts touch down. At this voltage the lever beam deflection is still small. As the voltage continues to increase, the plate is deforming, resting posts closer to the lever touch down and the lever edge of the plate moves down as well, acting on the lever and lifting the shutter up.

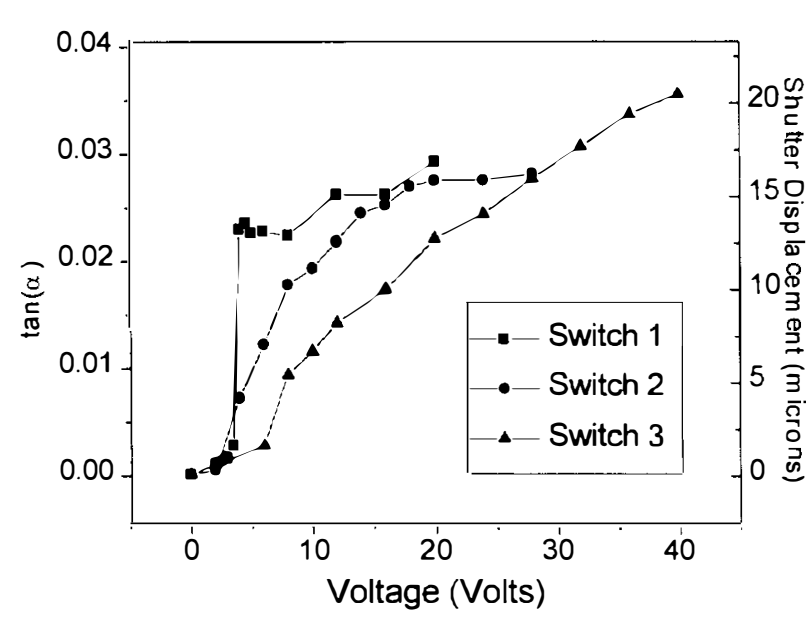

Figure 4. Lever tilt angle and shutter displacement as a function of actuator voltage. Switch I has a first generation lever design, Switches 2 and 3 have second generation levers with different flexure stiffness. These curves are defined by the stiffness of the plate and lever suspensions, pivot and capacitor geometry and lever length.

This zip-lock action was directly observed by looking at the movable plate shape with an interferometric profilometer. It also manifests itself in the displacement vs. voltage characteristic of the device: while for softer levers, (i.e. Switch 1) a snap-down voltage exists corresponding to the $1 / 3$ instability of a parallel-plate actuator, for stiffer lever mechanisms, (Switches 2 and 3 ) displacement is a smooth function of voltage, corresponding to the continuous zip-lock action. This mode of operation may also be beneficial in lowering the actuation voltage.

Our switches had the following optical characteristics: insertion loss in the transmit state including loss of two FC-PC connectors ranged from 0.81 to $3.26 \mathrm{~dB}$, return loss in the reflection state varied from 1.15 to $3.77 \mathrm{~dB}$, including the loss of two connectors. These insertion losses are comparable to the ones achieved with conventional opto-mechanical switches. Transmission isolation ranged between $38 \mathrm{~dB}$ to greater then 80 $\mathrm{dB}$ while reflection return loss varied from 15 to $25 \mathrm{~dB}$. The low return loss resulted from weak reflections in the fiber gap and might be improved by using angle-cleaved rather than antireflection-coated fibers. Less than $0.05 \mathrm{~dB}$ polarization-state induced variation of transmit-state insertion loss or reflect-state return loss was observed.

Fig. 5 shows light transmission as a function of the applied voltage for three different switch designs. The smooth, continuous switching action for Switches 2 and 3 seen on Fig. 5 make them particularly good candidates for use as variable attenuators with almost $80 \mathrm{~dB}$ of dynamic range [7]. 


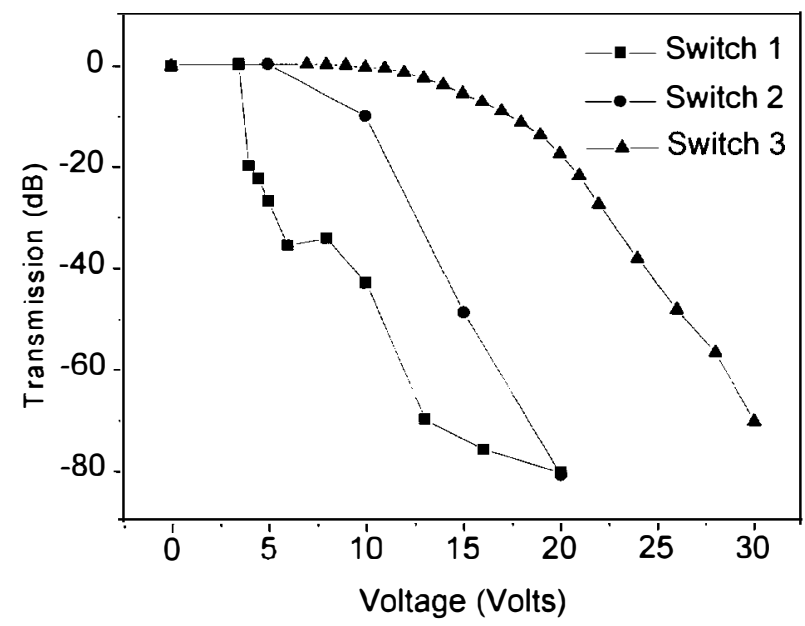

Figure 5. Switch transmission as a function of actuator voltage. Switches 2 and 3 may also be used as variable attenuators with high dynamic range

Fig. 6 and 7 show transmission as a function of time for two different switches under various square-wave applied voltages. From these data, the rise and fall times can be extracted, as well as switching delay time. defined as the time between the voltage step and the start of the optical switching action.

Switch opening times corresponding to a voltage change from a finite value to 0 are determined by the spring constants of the flexures returning the shutter to its initial position, the shutter inertia as well as the position of the shutter in the closed state.

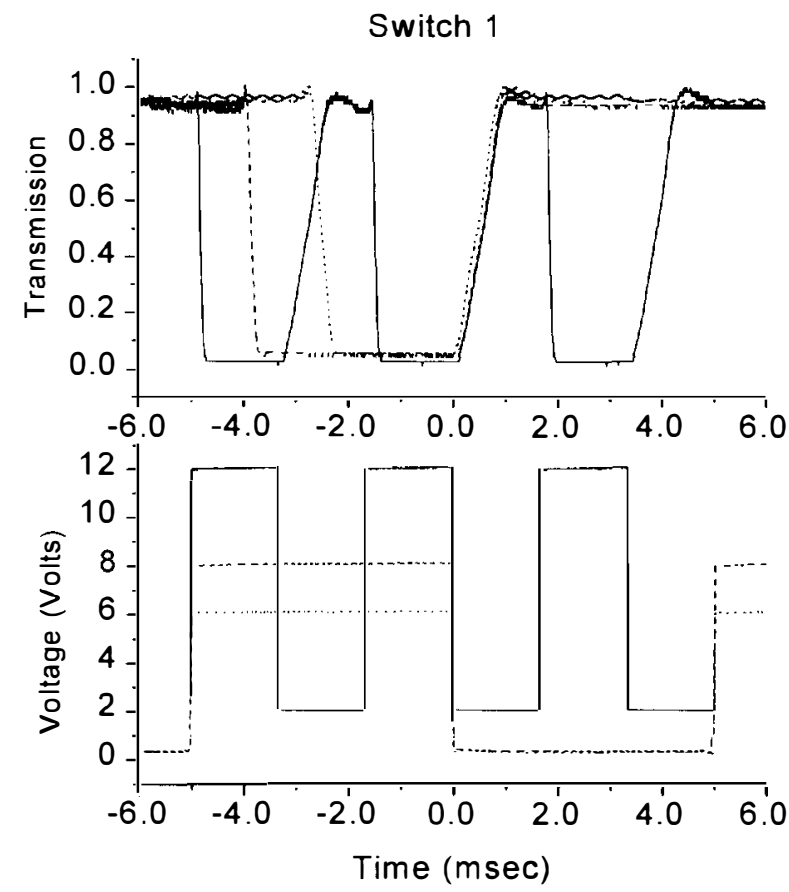

Figure 6. First generation, low voltage switch operation with different actuator voltages. Shutter closing delay and fall times are voltage dependent while shutter opening delay and rise times are not
These values are less then $150 \mu \mathrm{sec}$ delay and $890 \mu \mathrm{sec}$ rise for Switch 1 (softer flexures) and $65 \mu \mathrm{sec}$ delay and $45 \mu \mathrm{sec}$ rise time for Switch 2 (stiffer flexures). The opening delay time is due to the shutter edge placed initially above the light beam, blocking the light, and having to travel a finite distance down before letting the light through. The rise times correspond to the speed of the shutter edge crossing the beam. For Switch 1 the shutter position in the closed state is defined by the lever mechanism geometry and is voltage independent, while for Switch 2 the shutter is higher for larger actuation voltage resulting in longer switch delay time and shorter rise time.

The closing delay and fall times correspond to the voltage step from 0 to a finite value and the shutter moving up. They are determined by the distance the shutter has to travel before it starts blocking the beam, the actuator force accelerating it and its mass.

\section{Switch 2}

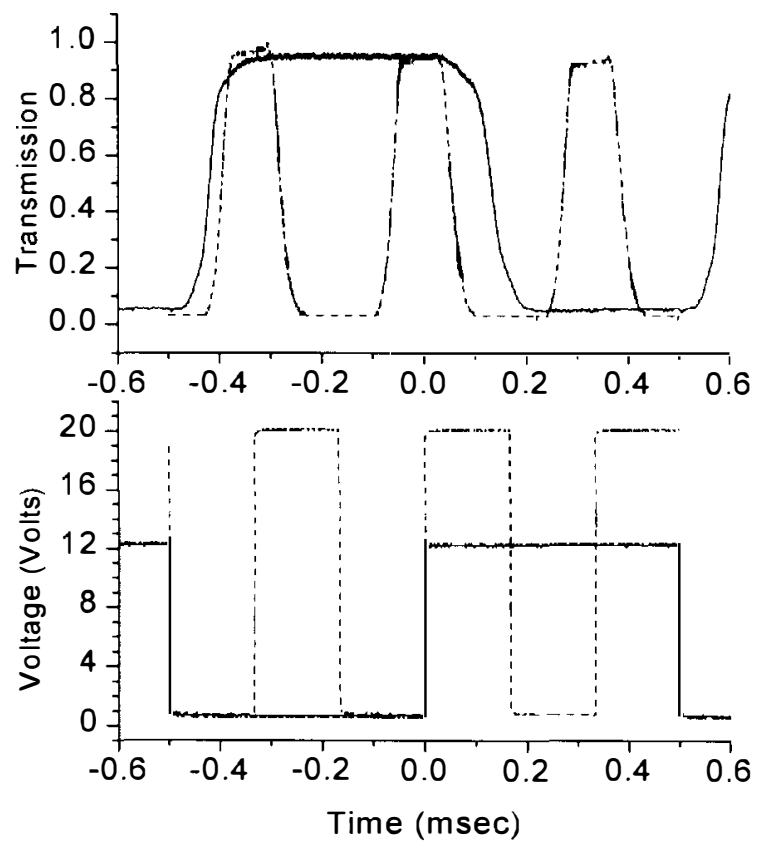

Figure 7. Second generation, fast switch operation. 10 times faster operation relative to $S$ witch I is achieved with twofold increase in actuation voltage.

In Fig.8, closing times are shown to monotonously decrease with increasing voltage, as the shutter is driven up faster by the larger electrostatic force. At large drive voltages the speed of the device is limited by the opening time. It is either voltage independent or increasing slightly and then saturating, as the position of the shutter under bias becomes defined by the actuator geometry. In addition, a tradeoff exists between the maximum switching speed and the minimum actuation voltage - stiffer flexures make the switching action faster but require larger actuation forces and correspondingly larger voltages.

Currently the effort is being made to further optimize the switch performance for higher speeds and lower actuation voltages by decreasing the shutter mass and improving the actuator design. In particular, first tests have been successful on the next generation lever mechanism where the friction point has been eliminated. To lower the actuation voltage thinner, more compliant flexures are also being considered. 


\section{REFERENCES}

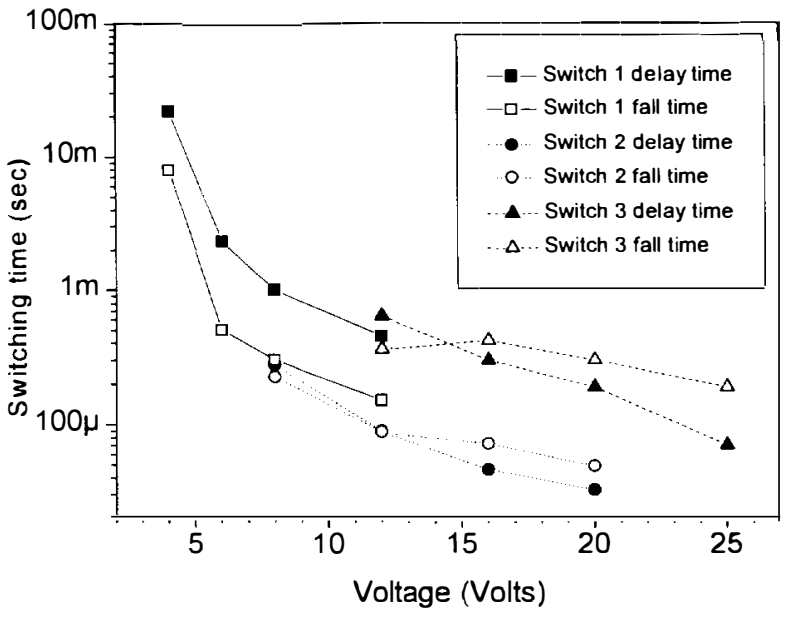

Figure 8. Switch closing time as a function of actuator voltage. Solid symbols - switch delay time. Open symbols - fall time. Delay time results from the shutter having to accelerate and travel some distance before it starts blocking the beam.

\section{CONCLUSION}

In summary, we have described a new Si-surfacemicromachined reflective optical switch suitable for use in reconfigurable lightwave network components including protection switches, optical cross-connects and WDM add/drop multiplexers. Having a movable thin gold-coated silicon vane in the switch enabled close placement of optical fiber endfaces to achieve excellent optical characteristics without additional lenses or collimators. These switches had a minimum insertion loss of $0.8 \mathrm{~dB}$. exhibited $\sim 2 \mathrm{~dB}$ return loss and $>80 \mathrm{~dB}$ transmission isolation in the reflect-state. The minimum actuation voltage was $4 \mathrm{~V}$ and with $20 \mathrm{~V}$ they were able to switch in $50 \mu \mathrm{sec}$. Very compact packaging was shown and quiescent power consumption of the electrostatic actuator was negligible. Two actuator geometries were investigated here and new geometries are being developed to optimize the drive voltage and switching times. The actuator design is amenable to other switch configurations, including $2 \times 2$ optical switches with a single actuator, and larger fabrics using arrays of devices.
1. H. Toshiyoshi and H. Fujita, "Electrostatic Micro Torsion Mirrors for an Optical Switch Matrix", Journal of Microelectromechanical Systems, vol. 5, no. 4, 231 (1996).

2. S.S. Lee, E. Motamedi and M.C. Wu, "Surface-Micromachined Free-Space Fiber Optic Switches With Integrated Microactuators for Optical Fiber Communication Systems", Transducers '97 Technical Digest, Vol. 1, p. 85.

3. E. Hashimoto, Y. Uenishi, K. Honoma and S. Nagaoka, Transducers'97 Technical Digest, Vol.1, p.331.

4. C. Marxer, C. Thio, M.-A. Gretillat, N. F. De Rooij, R. Bättig, O. Anthamatten, B. Valk, P. Vogel, "Vertical Mirrors Fabricated By Deep Reactive Ion Etching For Fiberoptic Switching Applications", Journal of Microelectromechanical Systems, vol. 6. no. 3, 277 (1997).

5. D. Koester, R. Majedevan, A.Shishkoff, and K. Marcus, MultiUser MEMS Processes (MUMPS) Introduction and Design Rules, rev. 4, 7/15/96, MCNC MEMS Technology Applications Center, Research Triangle Park, NC 27709

6. K. S. J. Pister, M. W. Judy, S. R. Burgett, and R. S. Fearing, "Microfabricated hinges", Sensors and Actuators A, vol. 33. no. 3, 249 (1992)

7. B. Barber et al., submitted to Electronics Letters. 